\title{
30
}

\section{VIRTUAL POWER PRODUCERS INTEGRATION INTO MASCEM}

Isabel Praça, Hugo Morais, Marílio Cardoso, Carlos Ramos, Zita Vale GECAD - Knowledge Engineering and Decision Support Group Institute of Engineering - Polytechnic of Porto

Porto, PORTUGAL

\{icp,hgvm,joc, csr,zav\}@isep.ipp.pt

\begin{abstract}
All over the world Distributed Generation is seen as a valuable help to get cleaner and more efficient electricity. Under this context distributed generators, owned by different decentralized players can provide a significant amount of the electricity' generation. To get negotiation power and advantages of scale economy, these players can be aggregated giving place to a new concept: the Virtual Power Producer. Virtual Power Producers are multitechnology and multi-site heterogeneous entities. Virtual Power Producers should adopt organization and management methodologies so that they can make Distributed Generation a really profitable activity, able to participate in the market. In this paper we address the integration of Virtual Power Producers into an electricity market simulator - MASCEM - as a coalition of distributed producers.
\end{abstract}

\section{INTRODUCTION}

The development of new low emission generation technologies (wind generation, solar cells, fuel cells, micro-turbines) leads us to rethink the location of a significant part of the production: distributed generators owned by decentralized players will provide a significant amount of the electricity generation.

With Distributed Generation (DG), electricity is produced near consumers' location and not transmitted over long distances. Thus, it is possible to get lower losses. Moreover, new generation technologies, mainly based on renewable resources, with environmental advantages are a key issue for sustainable development. Investments in this field are encouraged by a favorable regulatory framework and the equipment costs are more attractive every day. At this stage, the main technologies used are micro and hydro turbines, fuel cells, wind generation, and solar cells.

A deregulated market where every single low power rating generation unit sells its power on the market would be optimal for the whole community both economically and technically.

To get negotiation power and advantages of scale economy, these players can be aggregated giving place to a new concept: the Virtual Power Producer (VPP). VPPs are multi-technology and multi-site heterogeneous entities. VPPs should adopt organization 
and management methodologies so that they can make DG a really profitable activity, able to participate in these markets.

In order to operate in an efficient way, VPPs should have adequate decisionsupport tools. These must be based on the availability and processing of the required information and knowledge concerning producers and market operation. A successful achievement of VPPs' goals requires the use of a mix of adequate technologies for optimizing and supporting their activities.

Agents and multi-agent systems that adequately simulate electricity markets behavior are essential tools to gather knowledge to provide decision-support to strategic behavior.

MASCEM- Multi-Agent Simulator of Competitive Electricity Markets (Praça et al., 2003) was developed to study several negotiation mechanisms usually found in electricity day-ahead markets. In MASCEM market participants have strategic behavior and a scenario decision algorithm to support their decisions. With MASCEM several experiences have already been made, leading us to achieve some conclusions and define future developments. One of the most important goals of MASCEM is the simulation of several different electricity market mechanisms.

With VPP integration into MASCEM, VPP proposals can be effectively evaluated under scenarios considering several different market mechanisms and containing several other producers and consumer entities. With MASCEM, VPP can improve their strategies to face market.

\section{MASCEM OVERVIEW}

A Pool is a marketplace where electricity-generating companies submit production bids and their corresponding market prices, and consumer companies submit consumption bids. A Market Operator regulates the pool. The Market Operator uses a market-clearing tool to set market price and a set of accepted production and consumption bids for every hour. In Pools, an appropriate market-clearing tool is an auction mechanism.

Bilateral Contracts are negotiable agreements between two traders about power delivery and receipt. The Bilateral-Contract model is flexible; negotiating parties can specify their own contract terms.

The Hybrid model combines features of Pools and Bilateral Contracts (Shahidehpour et al., 2002). In this model, a Pool isn't mandatory, and customers can either negotiate a power supply agreement directly with suppliers or accept power at the established market price. This model therefore offers customer choice.

There are several entities involved in the negotiations; we propose a multi-agent model to represent all the involved entities and their relationships.

MASCEM multi-agent model includes: a Market Facilitator Agent, Seller Agents, Buyer Agents, Trader Agents, a Market Operator Agent and a System Operator Agent. Three types of markets are simulated: Pool Markets, Bilateral Contracts and Hybrid Markets.

The Market Facilitator is the coordinator of the market. It knows the identities of all the agents present in the market, regulates the negotiation process and assures the market is functioning according to the established rules. The first step agents' have to do is the registration at the Market Facilitator, specifying their market role and ser-vices. 


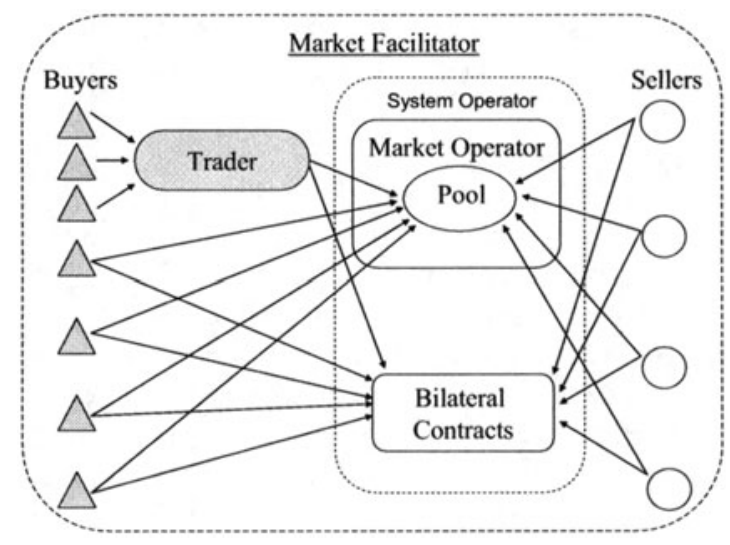

Figure 1 -- MASCEM multi-agent model

Seller and Buyer Agents are the two key players in the market. Sellers represent entities able to sell electricity in the market, e.g. companies holding electricity production units. Buyers may represent electricity consumers or even distribution companies. The user, who must also specify their intrinsic and strategic characteristics, defines the number of Sellers and Buyers in each scenario. By intrinsic characteristics we mean the individual knowledge related to reservation and preferred prices, and also to the available capacity (or power needs if it is a Buyer). By strategic characteristics we mean the strategies the agent will employ to reach its objectives.

Sellers will compete with each other, since they are all interested in selling all their available capacity and in obtaining the highest possible market quote. On the other hand, Sellers will cooperate with Buyers while trying to establish some agreement that is profitable for both. This is a very rich domain where it is possible to develop and test several algorithms and negotiation mechanisms for both cooperation and competition.

The System Operator Agent represents the responsible for the transmission grid and all the involved technical constraints. Every contract established, either through Bilateral Contracts or through the Pool, must first be communicated to the System Operator, who analyses its technical viability from the Power System point of view (e.g. feasibility of Power Flow to attend all needs).

The Market Operator Agent represents the responsible for the Pool mechanism. This agent is only present in simulations of Pool or Hybrid markets. The Market Operator will receive bids from Sellers, Buyers and Traders, analyze them and establish the marginal price and accepted bids. The process of determining the accepted bids is done according to the technical validation by the Sys-tem Operator, after, the Market Operator communicates to Sellers, Buyers and Traders the acceptance, or not, of their bids and, optionally, the market price.

The increase in competitiveness creates opportunities for many new players to enter the market; one of these players is the Trader. The introduction of this new entity allows liberalization and competition in the electricity industry to be developed and simplifies the way the whole process works with producers and customers on the market and the relationship with the Market Operator. This entity 
participates in the market on behalf of customers. It is an intermediary between them, who delegate on the Trader the purchasing of their needs, and the suppliers. The increasing role of this type of entity turn it an important feature of our simulator.

\section{MASCEM NEGOTIATION}

On the basis of the results obtained in a negotiation period Sellers, Buyers and Traders revise their strategies for the next period. Seller, Buyer and Trader Agents have strategic behavior to define their desired price. These agents have timedependent strategies, to change the price according to the remaining time until the end of the negotiation period; and behavior-dependent strategies, to define the next period price according to the results obtained in the previous ones.

MASCEM implements four types of strategies to change the price during a negotiation period: Determined, Anxious, Moderate and Gluttonous. The difference between these strategies is the time instant at which the agent starts to modify the price and the amount it changes. Although time-dependent strategies are simple to understand and implement (Morris et al., 2003), they are very important since they allow the simulation of important issues such as: emotional aspects and different risk behaviors. For ex-ample: an agent using a Determined Strategy is a risk indifferent one; while Gluttonous agents exhibit the behavior more risk disposable, since they maintain the same price until very close to the end of the negotiation period, taking the risk of not selling.

To adjust price between negotiation periods, also referred as behavior-dependent strategies, two different strategies were implemented: one called Composed Goal Directed and another called Adapted Derivative Following, see details in (Praça et al., 2005). These are important strategies that use the knowledge obtained with past experiences to de-fine bid prices for next periods.

To obtain an efficient decision support, Seller and Buyer agents also have the capability of using the Scenario Analysis Algorithm.

This algorithm provides a more complex support to develop and implement dynamic pricing strategies since each agent analyses and develops a strategic bid, for the next period, taking into account not only their previous results but also other players results and expected future reactions. It is particularly suitable for markets based on a Pool or for Hybrid markets, to support Sellers, Buyers and Traders decisions for proposing bids to the Pool and accepting or not a bilateral agreement. The algorithm is based on analyzing several bids under different scenarios, constructing a matrix with the obtained results and applying a decision method to select the bid to propose.

Each agent has historical information about market behavior and about other agents' characteristics and behavior. To get warrantable data, each agent uses techniques based on statistical analysis and knowledge discovery tools, which analyze the historical data. With the information gathered agents can build a profile of other agents based on their expected proposed prices, limit prices, and capacities. With these profiles, and based on the agent own objectives, several scenarios, and the possible advantageous bids for each one, are defined. The agent should analyze the incomes that result from bidding its limit, desired prices, and competitive prices - those that are just slightly lower (or higher, in the Buyer's case) than its competitors' prices. 
We call a play to a pair bid-scenario. After defining all the scenarios and bids, market simulation is applied to build a matrix with the expected results for each play.

The matrix analysis with the simulated plays' results is inspired by the game theory concepts for a pure-strategy two-player game, assuming each player seeks to minimize the maximum possible loss or maximize the minimum possible gain (Fudenberg and Tirole, 1991).

A Seller-like an offensive player - will try to maximize the minimum possible gain by using the MaxiMin decision method. A Buyer-like a defensive playerwill select the strategy with the smallest maximum payoff by using the MiniMax decision method. In Buyers' matrix analyses, they select only situations in which they can fulfill all their consumption needs. They avoid situations in which agents will accept reduced payoff but can't satisfy their consumption needs completely.

The analysis of each period's results will update the agent's market knowledge and the scenarios to study. After each negotiation period, instead of considering how they might increase, decrease, or maintain their bid, agents use knowledge rules that restrict modifications on the basis of other agents' expected behavior.

The knowledge rules update agents' bids in each scenario, but the number of scenarios remains the same. If at the end of a negotiation period the agent concludes — by analyzing market results - that it incorrectly evaluated other agents' behavior, it will fix other agents' profiles on the basis of the calculated deviation from real results.

\section{VIRTUAL POWER PRODUCERS}

The aggregation of Distributed Generation plants gives place to the new concept of Virtual Power Producers (VPP). VPPs are multi-technology and multi-site heterogeneous entities, being relationships among aggregated producers and among VPPs and the remaining Electricity Market (EM) agents a key factor for their success. An aggregating strategy can enable owners of Distributed Generation to gain technical and commercial advantages, making profit of the specific advantages of a mix of several generation technologies and overcoming serious disadvantages of some technologies.

Any type of generation unit or load may be included: wind turbines, photovoltaic, mini turbines, micro-turbine, fuel cells, energy storage units, non-controllable loads, controllable loads etc. The typical size of single distributed energy resource units may range from a few $\mathrm{kW}$ to some $\mathrm{MW}$.

In the scope of a VPP, aggregated producers (AP) can make sure their generators are optimally operated and that the power that is not consumed in their installation has good chances to be sold on the market. At the same time, VPPs will be able to commit to a more robust generation profile, raising the value of non-dispatchable generation technologies.

Under this context, VPPs can ensure secure, environ-mentally friendly generation and optimal management of heat, electricity and cold and optimal operation and maintenance of electrical equipment, including the sale of electricity to the EM. VPPs should adopt organization and management methodologies so that they can make Distributed Generation a really profitable activity. 
VPPs must be flexible enough to use the advantages of its resources (e.g. marketbased environmental value in the form of pollution and/or carbon credits, renewable energy credits) and overcoming their problems and limitations.

VPPs must identify the characteristics of each of the AP and try to optimize the selling activity so that each associate delivers the biggest possible amount of energy. However, this is not simple due to uncertainty of generation associated with the technologies that depend from natural resources such as wind, sun, waves or water flows.

So, in order to have VPP able to coexist with other market agents, it is necessary that it gets profits and that has credibility in the EM. This context must be considered in VPPs organization and operation methodologies as their goal is to optimize their APs' profits in this market.

A successful achievement of VPPs' goals requires the use of a mix of adequate technologies for optimizing and supporting their activities. Under this scope agents and multi-agent systems are important technologies to adequately simulate EM behavior and gather knowledge to provide decision-support to strategic behavior.

Taking into account the already described MASCEM characteristics, it can be a valuable framework to test VPP functioning under different market mechanisms and concerning different market strategic behavior. But, how can VPP be integrated into MASCEM model?

\subsection{Coalitions in Multi-Agent Systems}

Coalition formation is the coming together of a number of distinct, autonomous agents that agree to coordinate and cooperate, acting as a coherent grouping, in the performance of a specific task. Such coalitions can improve the performance of the individual agents and/or the system as a whole. It is an important form of interaction in multi-agent systems.

It has been advocated in e-commerce (where buyers may pool their requirements in order to obtain bigger group discounts), in grid computing (where multiinstitution virtual organizations are viewed as being central to coordinated resource sharing and problem solving), and in e-business (where agile groupings of agents need to be formed in order to satisfy particular market niches). In all of these cases, the formation of coalitions aims to in-crease the agents' abilities to satisfy goals and to maximize their individual or the system's outcomes.

Most work on coalition formation in Multi-Agent systems and game theory has focus on payoff distribution, where it is usually assumed that a coalition structure has been formed, and the question is then how to divide the payoff so that the coalition structure is stable. In this con-text, many solutions have been proposed based on different stability concepts. Transfer schemes have also been developed to transfer non-stable payoff distributions to stable ones (while keeping the coalition structure un-changed).

Recently, research is giving more attention to the coalition structure generation (Sandholm et al., 1999) (Dang and Jennings, 2004). The work of Shehory and Kraus (Shehory and Kraus, 1996) considers a somewhat broader environment, where the coalitions can be overlapped but the complexity is reduced by limiting the size of the coalitions.

Some other researchers address both coalition structure generation and payoff distribution in competitive environments. Ketchpel (Ketchpel, 1994) presents a 
coalition formation method with cubic running time in the number of agents, but his method can neither guarantee a bound from the optimal nor stability. Shehory and Kraus's protocol guarantees that if the agents follow it, certain stability (kernelstability) is met. In the same paper, they also present an alternative protocol that offers a weaker form of stability with polynomial running time. However, in both cases, no bound from the optimal is guaranteed.

More recent research in coalition formation area has also begun to pay attention to dynamic environments, where agents may enter or leave the coalition formation process and many uncertainties are present (e.g. the coalition value is not fixed, but it is context-based (Klush and Gerber, 2002)).

\subsection{VPP Coalition Formation in MASCEM}

A negotiation mechanism regarding coalition formation under the scope of a VPP is in fact being included in MASCEM, and strategies will be developed considering the three phases of a coalition's formation process.

VPP needs to have an adequate knowledge of each potential aggregated producer characteristics. We are working on a multi-criteria negotiation protocol.

Some of the most important characteristics are:

- Nominal Power: the sum of nominal power in-stalled in each producer;

- $\quad$ Available Power: the power a VPP can buy to the producer;

- Overload Power: some units may produce overload power for limited periods. The VPP may use this power in critical situations;

- Equipment characteristics: information concerning producers' equipment allows the VPP to know the power characteristic, reliability, maintenance periods, lifetime, relation with external factors, possible variations of the energy price in function of the cost of the primary resources, etc.

- Operating limits: for the units which are dependent from natural resources, it is possible that the primary resource must be below or above of equipment operating limits. This must be considered in risk analysis in the generation forecast. Usually when the resources forecast is near to the minimum machines operating limit the risk is small, but when they are near to the maximum limit the risk can be enormous;

- Grid connection characteristics: This is an important aspect if it is necessary to pay the losses in the lines; also the existence of two or more producers connected to the same electric substation should be considered; etc;

- Historical generation data: the availability of historic generation data can enable the VPP to get useful forecasting tools.

These issues must be carefully analyzed before a new producer enters the coalition. A multi-criteria decision function is being developed. This function must be a dynamic one, since the characteristics weights depends on the lack of already aggregated producers.

Regarding VPP market participation, the same market interface as Seller or Buyer agents will be used. However, there are some preliminary steps to define its proposals and to divide market results among VPP members. First all the capacity available from the different aggregated producers must be gathered, to establish the electricity amount to trade on the market, and the different production costs analyzed to define the interval for acceptable proposals. This means VPP agents will 
have a utility function that aggregates all the involved units' characteristics. The analysis of received proposals will be done according to each unit capabilities and costs.

Important is the use of previsions, such as climatic ones, to update the database of aggregated producers to forecast more and more efficiently the energy they will be able to provide to the VPP.

\section{ACKNOWLEDGEMENTS}

The authors would like to acknowledge FCT, FEDER, POCTI, POSI, POCI and POSC for their support to R\&D Projects and GECAD Unit.

\section{REFERENCES}

1. Dang V. D., Jennings N. Generating coalition structures with finite bound from the optimal guarantees. Proceedings 3rd International Conference on Autonomous Agents and Multi-Agent Systems, Nova York, E.U.A., 2004; 564-571.

2. Fudenberg D., Tirole J. Game Theory. MIT Press, 1991.

3. Ketchpel S. P. Forming coalitions in the face of uncertain rewards. Proceedings of the Twelfth National Conference on Artificial Intelligence 1994; 414-419.

4. Klusch M., Gerber A. Dynamic coalition formation among rational agents. IEEE Intelligent Systems $2002 ; 17: 3 ; 42-47$.

5. Morris J., Greenwald A., Maes P. Learning Curve: A Simulation-based Approach to Dynamic Pricing, Electronic Commerce Research. Special Issue on Aspects of Internet Agent-based E-Business Systems. Kluwer Academic Publishers, 2003; 3:245-276.

6. Praça I., Ramos C., Vale Z., Cordeiro M. MASCEM: A Multiagent System that Simulates Competitive Electricity Markets. IEEE Intelligent Systems, 2003; 18:6; 54-60.

7. Praça I., Ramos C., Vale Z., Cordeiro M. Intelligent Agents for Negotiation and Game-based Decision Support in Electricity Markets. Engineering Intelligent Systems Journal, Special Issue "Intelligent Systems Application to Power Systems", 2005; 13:2; 147-154.

8. Sandholm T., Larson K., Andersson M., Shehory O., Tohme F. Coalition structure generation with worst case guarantees. Artificial Intelligence, 1999; 111: 1-2; 209-238.

9. Shahidehpour M., Yamin H., Zuyi Li Market Operations in Electric Power Systems: Forecasting, Scheduling and Risk Management. John Wiley \& Sons, 2002.

10. Shehory O., Kraus S. A kernel-oriented model for coalition-formation in general environments: Implementation and results. Proceedings of the Thirteenth National Conference on Artificial Intelligence, 1996; 134-140. 